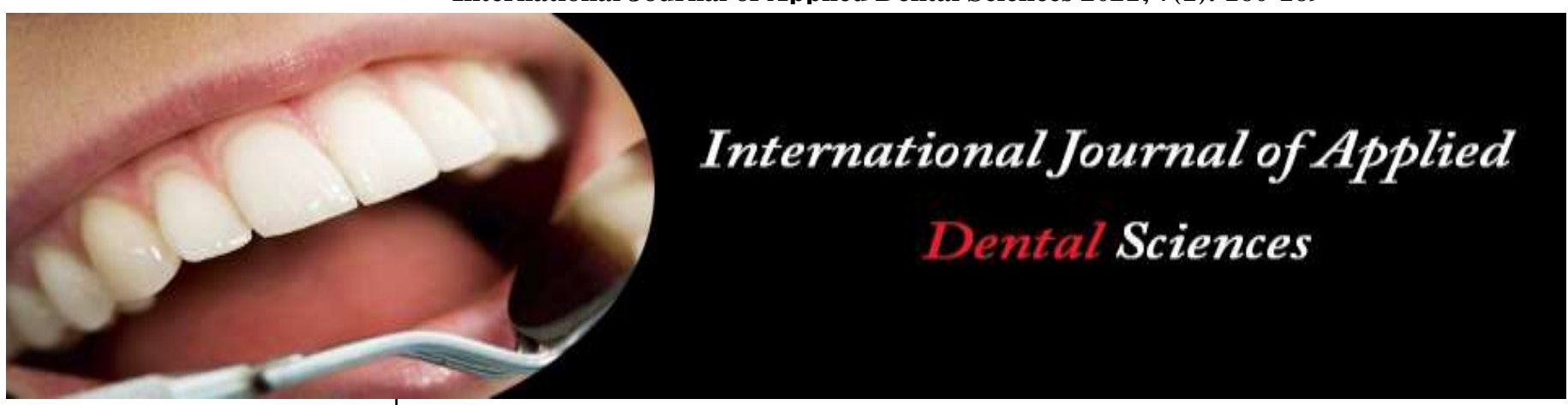

ISSN Print: 2394-7489

ISSN Online: 2394-7497

IJADS 2021; 7(1): 160-169

(C) 2021 IJADS

www.oraljournal.com

Received: 09-11-2020

Accepted: 18-12-2020

Dr. Jaishree Tukaram Kshirsagar Professor, Department of Periodontics, Tamilnadu Government Dental College and Hospital, Chennai, Tamil Nadu, India

Dr. Kokhila S

Post Graduate Student, Department of Periodontics, Tamilnadu Government Dental College and Hospital, Chennai, Tamil Nadu, India

\section{Standard dental operating protocol in COVID-19 pandemic}

\section{Dr. Jaishree Tukaram Kshirsagar and Dr. Kokhila S}

DOI: $\underline{\text { https://doi.org/10.22271/oral.2021.v7.i1c.1131 }}$

Abstract

Coronavirus belong to a large family of virus called Coronaviridae predominantly affects respiratory system. Virus that cause COVID-19, spreads mainly by droplet infection from oral or nasal secretions. Clinical presentation starts with fever, headache, cough, malaise and gastro- intestinal symptoms. Loss of taste and smell have been reported. Most of the patients recover with milder symptoms, few reported organ dysfunction and death. Dentistry is facing its toughest time with the spread of COVID-19 and the dental practice has been affected significantly in this pandemic. Most dental procedure require close contact with patient's oral cavity and treatment interventions generate aerosol, chance of cross infection is more in a clinical set up. Dentists and the patients have to follow strict measures to prevent the spread of pandemic. All patients visiting a dental office must be treated with due precautions.

Keywords: Dental protocol, fomites, tele dentistry, pandemic

\section{Introduction}

Coronavirus outbreak has created fear and panic among people all over the world and it is a breaking news with updates every day. On December 2019, cluster of pneumonia cases with unknown etiology were reported in Wuhan, China. WHO declared the outbreak a Public Health Emergency of International Concern on January 30, $2020^{[1]}$. On February 11,2020 WHO collaborated with World Organisation for Animal health, Food and Agricultural Organisation of the United Nations named the disease as COVID-19, abbreviation for Coronavirus disease $2019^{[2]}$. Previously called novel coronavirus 2019 now WHO in collaboration with International Committee on Taxonomy of virus, ICTV named it as SARS COV-2, Severe Acute Respiratory Syndrome Coronavirus-2 ${ }^{[3]}$. On March 11, 2020 WHO announced that COVID-19 can be characterized as a Pandemic ${ }^{[4]}$. All over the world, Health care professionals are at increased risk of acquiring COVID-19. As dental professionals are working in close proximity to oropharyngeal region, the risk of exposure to Coronavirus is high. Face to Face communication between the dentist and patients puts both at risk of cross infection.

\section{Structure of coronavirus}

Coronavirus belong to the family Coronaviridae and sub family Coronaviridae. Four genera, namely Alpha coronavirus, Beta coronavirus, Gamma coronavirus, Delta coronavirus exist ${ }^{[5]}$. Coronaviridae species are widespread among animals causing mild - respiratory infection, Coronavirus are single stranded envelope RNA virus, diameter ranges from $60-140 \mathrm{~nm}{ }^{[6]}$. They are named after presence of pointed structure on surface, resembling crown [7]. Coronavirus species are circulating in bats, subspecies Rhinolophus [8, 9]. Until 2002, Coronavirus were considered only minor pathogens of humans, SARS-COV Outbreak occurred in $2012^{[10]}$. MERS-COV Middle East Respiratory Syndrome Coronavirus caused infection in $2014^{[11,12]}$.

\section{Dr. Kokhila S}

Post Graduate Student,

Department of Periodontics,

Tamilnadu Government Dental College and Hospital, Chennai,

Tamil Nadu, India 


\section{Classification of coronavirus}

Table 1: Coronavirus can be classified ${ }^{[13]}$ as

\begin{tabular}{|c|c|}
\hline Human coronavirus & Incubation period \\
\hline $229 \mathrm{E}$ & $2-5$ days \\
\hline OC43 & $2-5$ days \\
\hline NL63 & $2-4$ days \\
\hline HKU1 & $2-4$ days \\
\hline SARS-CoV & $2-11$ days \\
\hline MERS-CoV & $2-13$ days \\
\hline 2019-nCoV & $1-14$ days \\
\hline
\end{tabular}

\section{Transmission mode}

Possible modes of transmission include Contact, Droplet, Airborne, Fomite, Fecal -oral, Blood borne, Mother to Child and Animal to Human transmission ${ }^{[14]}$. Respiratory secretion (or) droplets expelled by infected individuals can contaminate surfaces and objects creating fomites (contaminated surfaces) [15].

\section{Clinical presentation}

Disease expression varies from people to people, in terms of age, gender and underlying comorbid illness. The clinical picture varies from country to country. Most common symptoms are fever, cough, myalgia or tiredness, headache, Diarrhea and some people have reported loss of smell and taste ${ }^{[16]}$. Mild respiratory infections occur in about $80 \%$ of those infected; about $50 \%$ will have pneumonia, another $15 \%$ of patients develop severe illness, while 5\% need critical care treatment ${ }^{[17]}$.

\section{Diagnosis}

Confirmation of COVID-19 is based on the detection of unique sequence of virus RNA by NAAT (Nucleic Acid Amplification Test) technology such as Real Time Reverse Transcriptase polymerase chain reaction (rRT-PCR) ${ }^{[18]}$. Chest $\mathrm{X}$ ray and CT finding may be helpful in diagnosis of COVID19. Non-contact thermal scanning sensor is routinely used in public places. WHO does not recommend the use of Antibody -detecting rapid diagnostic test for patient care but encourages the continuation of work to establish their usefulness in disease surveillance and epidemiologic purpose ${ }^{[19]}$.

\section{Dental health care set up}

Dentist are constantly exposed to body fluids such as saliva and blood during dental procedure. Saliva is a known mode of transmitting infected droplets. Dentist are in danger of getting COVID-19 infection.

\section{Tele dentistry in COVID-19}

Tele dentistry is defined as the provision of real time and offline dental care such as diagnosis, treatment planning, consultation and follow-up through electronic transmission from different sites ${ }^{[20]}$. In this wide spread pandemic, advanced technology help us to manage patients who needs urgent dental care. All appointments and consultations are fixed through Tele dentistry. Patients who need intervention will get an appointment based on their clinical presentation. Appointments are scheduled appropriately to avoid crowding in a hospital environment. Attenders accompanying the patients shouldn't be encouraged, unless there is a valid reason.

\section{Patient arrival}

Sanitization and Social distancing norms are followed through the entire procedure. Patients are advised to fill their complete contact details and COVID-19 Screening form given by CDC (figure1) ${ }^{[21]}$. Complete medical history is evaluated. Dental Assistant should document the patient body temperature using Non- contact mode thermal sensor. Patient who has fever (or) susceptive of COVID-19, has been referred to Health care system. Patients are advised to wear a mask and hand sanitization is strictly followed.

\section{Waiting room}

Adequate ventilation and Social distancing norms should be strictly followed while waiting for the consultation. Appointments for patients should not overlap. Poster displaying Hand hygiene importance, cough etiquette, social distancing, COVID-19 Symptoms are made available for patient education.

\section{Disinfection and cleaning protocol in hospital}

Environmental surroundings inside hospital are likely to get highly contaminated with COVID-19, as medical procedure are carried out regularly. These contaminated surface should be thoroughly cleaned to remove dirt and organic load and then disinfected flowingly. Studies have evaluated the persistence of the COVID-19 on different surfaces, found that virus remained viable up to 1 day on cloth and wood, up to 2 days on glass, 4 days on stainless steel and plastic and up to 7 days on the outer layer of a medical mask ${ }^{[22]}$.

\section{Standard operating procedures (Sop) for cleaning and disinfection}

WHO have mentioned the cleaning and disinfecting protocols ${ }^{\text {[22] }}$ (table 2).

\section{Chemicals such as}

1. Ethanol 70 to $90 \%$

2. Chlorine - based products (Hypochlorite) at $0.1 \%$ (1000ppm) for general environmental disinfection

3. $0.5 \%$ (5000ppm) Hypochlorite for blood and body fluid large spills

4. Hydrogen peroxide

Fresh solution should be prepared on a daily basis. Proper concentration and volume of the disinfectant should be strictly prepared following the manufacturer's guidelines. Cleaning should progress from cleanest surface to dirtiest area, from higher level to lower place, so that debris may fall on the floor and cleaned last.

\section{Clinical set up}

Health care professionals (HCP) should follow safety precautions to avoid getting infection, as patients in incubation period or asymptomatic carriers may spread the disease to dentist. Dentist are one among the high risk category group under the health care professionals ${ }^{[23]}$. 
TODAY'S DATE:

\section{CDC FACILITIES COVID-19 SCREENING}

\section{Accessible version available at $h t t p s: / / w w w . c d c: g o v / s c r e e n i n g /$}

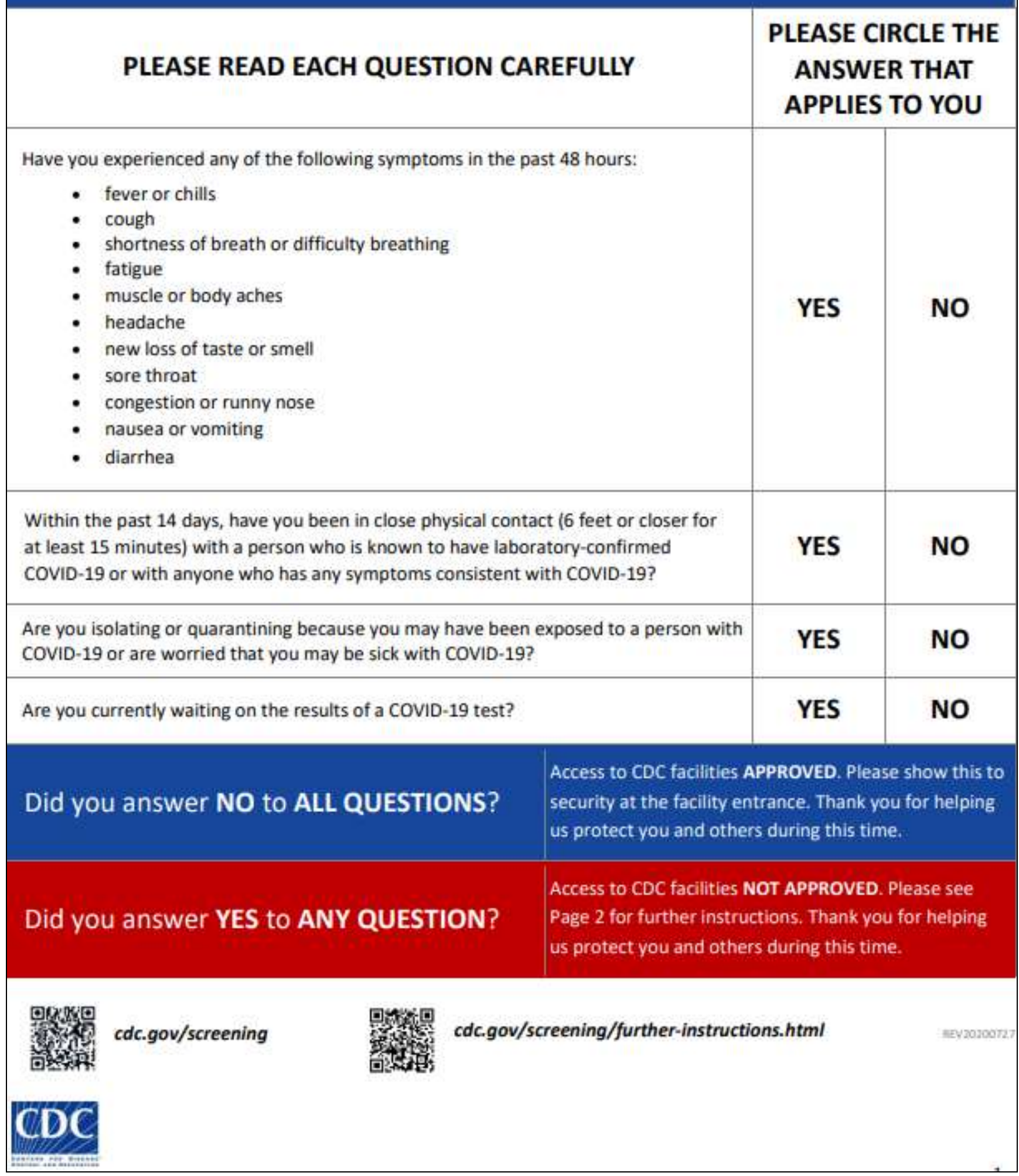

Fig 1: CDC Facilities COVID 19 screening 


\begin{tabular}{|c|c|c|}
\hline Patient area & Frequency ${ }^{\circ}$ & Additional guidance \\
\hline Screening/triage area & At least twice daily & - Focus on high-touch surfaces, then floors (last) \\
\hline $\begin{array}{l}\text { Inpatient rooms I cohort - } \\
\text { occupied }\end{array}$ & $\begin{array}{l}\text { At least twice daily, preferably three times } \\
\text { daily, in particular for high-touch surfaces }\end{array}$ & $\begin{array}{l}\text { - Focus on high-touch surfaces, starting with shared/common } \\
\text { surfaces, then move to each patient bed; use new cloth for } \\
\text { each bed if possible; then floors (last) }\end{array}$ \\
\hline $\begin{array}{l}\text { Inpatient rooms - unoccupied } \\
\text { (terminal cleaning) }\end{array}$ & Upon discharge/transfer & $\begin{array}{l}\text { - Low-touch surfaces, high-touch surfaces, floors (in that order); } \\
\text { waste and linens removed, bed thoroughly cleaned and } \\
\text { disinfected }\end{array}$ \\
\hline $\begin{array}{l}\text { Outpatient I ambulatory care } \\
\text { rooms }\end{array}$ & $\begin{array}{l}\text { After each patient visit (in particular for } \\
\text { high-touch surfaces) and at least once } \\
\text { daily terminal clean }\end{array}$ & $\begin{array}{l}\text { - High-touch surfaces to be disinfected after each patient visit } \\
\text { - Once daily low-touch surfaces, high-touch surfaces, floors (in } \\
\text { that order); waste and linens removed, examination bed } \\
\text { thoroughly cleaned and disinfected }\end{array}$ \\
\hline Hallways / corridors & At least twice daily ${ }^{b}$ & $\begin{array}{l}\text { - High-touch surfaces including railings and equipment in } \\
\text { hallways, then floors (last) }\end{array}$ \\
\hline Patient bathrooms/ toilets & $\begin{array}{l}\text { Private patient room toilet: at least twice } \\
\text { daily } \\
\text { Shared toilets: at least three times daily }\end{array}$ & $\begin{array}{l}\text { - High-touch surfaces, including door handles, light switches, } \\
\text { counters, faucets, then sink bowls, then toilets and finally floor } \\
\text { (in that order) } \\
\text { - Avoid sharing toilets between staff and patients }\end{array}$ \\
\hline
\end{tabular}

Recommends use of easily decontaminated physical barriers, local exhaust ventilation to capture and remove aerosols generated during treatment; and use of directional airflow to remove workplace hazards.

Recommends strategies such as telephone triage and teledentistry to determine the need for care. Within the office, screening for signs and symptoms, limiting the number of people in the treatment area, and minimizing aerosol-generating procedures used in dental treatment.

\section{Dental emergency}

Patients with Acute Oro dental Infections, Swelling and bleeding, Space Infections in Oro-Facial region, severe pain not manageable with Analgesics, Trauma to the dentition, Trauma involving facial bones require urgent or emergency dental intervention. If not manageable by General Dentist patient should be referred to higher speciality with precautions.
Patients with dental emergencies suspected of COVID-19, should be referred to COVID specialized oral health services, where appropriate urgent or emergency oral health care interventions are provided. Home visit by a dedicated oral health care team following strict infection prevention and control measures as locally prescribed could also be provided. The following approaches to treatment are recommended:

\begin{tabular}{|c|c|}
\hline Conditions & Various Treatment Approaches \\
\hline $\begin{array}{c}\text { Dent alveolar or periodontal abscess, acute } \\
\text { episodes of pain }\end{array}$ & Incision and drainage, antibiotic therapy \\
\hline Bleeding Gums, Gingivitis & Hand scaling and cleaning, antiseptic mouth rinse \\
\hline Pain due to acute periodontitis & Under local anesthesia, hand scaling, antibiotic therapy, antiseptic mouth rinse \\
\hline Acute pain due to carious tooth & $\begin{array}{c}\begin{array}{c}\text { Devitalisation of deep and open carious lesion with hand excavation and temporary } \\
\text { restoration }\end{array} \\
\end{array}$ \\
\hline Extensive carious tooth & Dental extraction only if non-restorable \\
\hline Fractured teeth & $\begin{array}{l}\text { Under local anesthesia, removal of the broken part or non-invasive restoration, } \\
\text { splinting if necessary }\end{array}$ \\
\hline Broken orthodontic appliances & Removal and fixation of the appliances \\
\hline Broken denture or prosthesis & Relining (Laboratory Repair) \\
\hline Acute pain due to mobile tooth & Splinting if prognosis is good a traumatic extraction \\
\hline Pericoronitis & Antibiotic therapy debridement of the flap antiseptic mouth rinse \\
\hline Apthous Ulcer & Topical analgesics antiseptic mouth rinse nutrition supplementation \\
\hline
\end{tabular}

Dental emergencies exacerbate in a short period of time, need immediate treatment care. Rubber dams and high-volume saliva ejectors can minimize aerosol generation or spatter in dental procedures. Face shields and goggles are essential during treatment with use of high or lowspeed drilling. During the pandemic, a carious tooth with symptomatic irreversible pulpitis is treated under local anaesthesia with the usage of rubber dam isolation and a high-volume saliva ejector, pulp devitalization procedure is performed to reduce the pain. It is recommended to rinse the wound slowly and use the saliva ejector to avoid spraying. Life-threatening cases should be admitted to the hospital immediately, and chest CT should be prescribed if available to exclude suspected infection because the RT-PCR test is time-consuming. 


\section{Aerosol Generating Procedures (AGPS)}

\section{Definition}

All clinical procedures that use spray generating equipment such as three-way air/water spray, dental cleaning with ultrasonic Scaler and polishing; periodontal treatment with ultrasonic Scaler; any kind of dental preparation with high or low-speed hand-pieces; direct and indirect restoration and polishing; definitive cementation of crown or bridge; mechanical endodontic treatment; surgical tooth extraction and implant placement ${ }^{[24]}$.
Pratice Four Handed Dentistry ${ }^{[25]}$, Use of high-speed suction and rubber dam are advised. Use of appropriate PPE including a fit tested N95 or FFP2 respirator, or higher is adviced during AGPs.

Guidelines for personal protective Equipment for health care professional

OSHA recommended

\section{OSHA PPE recommendations when providing dental treatment to well patients during the COVID-19 pandemic}

\begin{tabular}{|l|l|}
\hline $\begin{array}{l}\text { Treatment not involving aerosol-generating } \\
\text { procedures }\end{array}$ & $\begin{array}{l}\text { Treatments that may or are known to generate } \\
\text { aerosols }\end{array}$ \\
\hline $\begin{array}{l}\text { - Work clothing, such as scrubs, lab coats, } \\
\text { and/or smocks, or a gown }\end{array}$ & - Gloves \\
- Gloves & - Gown \\
- Eye protection (e.g. googles, face shield) & - Eye protection (e.g., googles, face shield) \\
- Face masks (e.g. surgical mask) & $\begin{array}{l}\text { flOSH-certified, disposable N95 filtering } \\
\end{array}$
\end{tabular}

Very high risk category includes treatment generating aerosol on known or suspected COVID-19 patients. High risky group includes treatment providing emergency dental care, not involving aerosol-generating procedures, to a known or suspected COVID-19 patient. Medium risky group are those who provide urgent or emergency dental care, not involving aerosol-generating procedures, to well patients (i.e., to members of the general public who are not known or suspected COVID-19 patients). Low risk category performing administrative tasks in non-public areas.

\section{WHO have given guidelines regarding the usage of Personal Protective Equipment ${ }^{[26]}$}

Table 3: Personal Protective Equipment must be used and that PPE differs depending on whether the work involves providing well patient care or care to a patient with suspected or confirmed COVID-19. "Dentistry works must use proper PPE when exposed to patients.

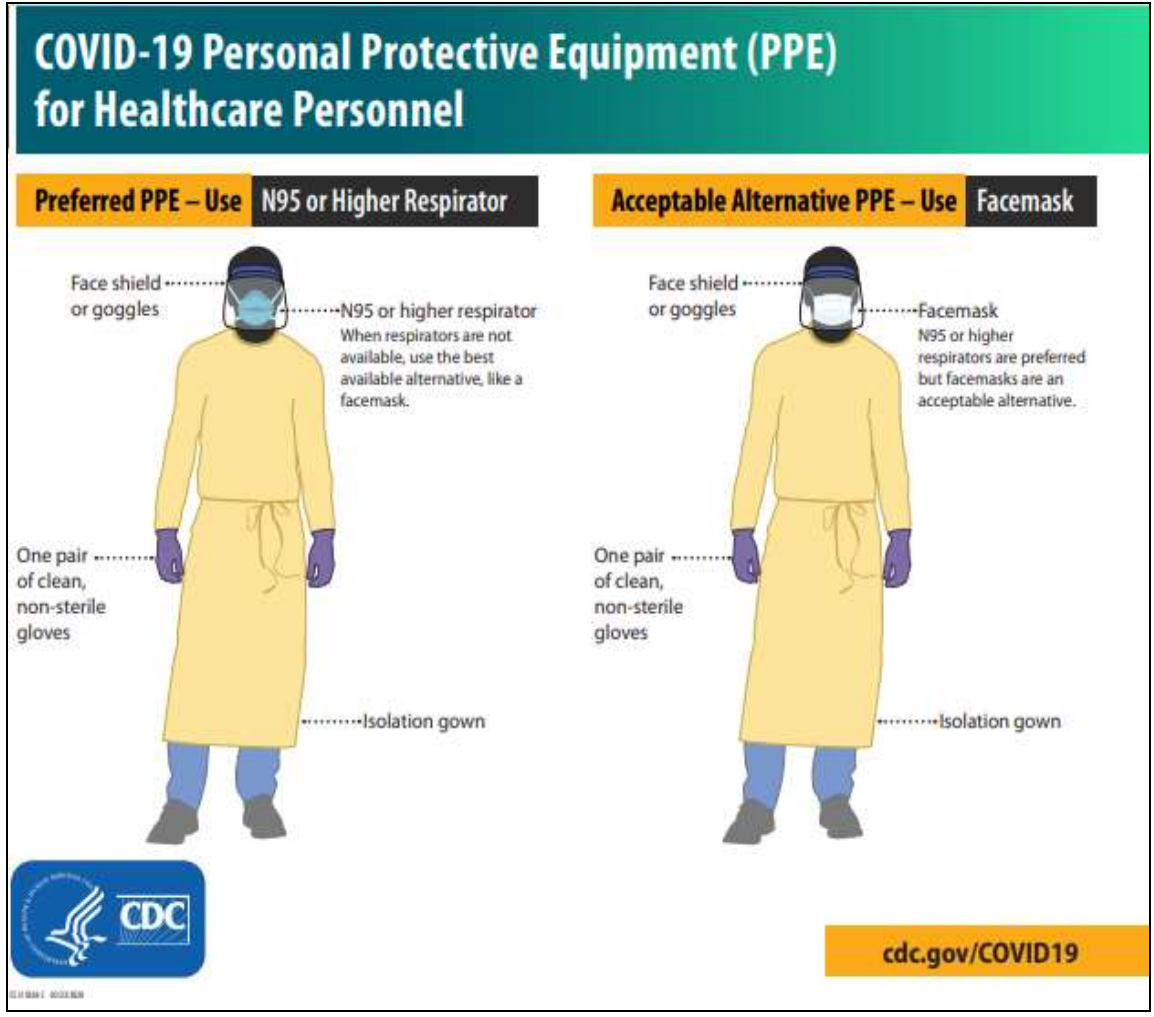


My Five Moments for Hand Hygiene principle given by WHO ${ }^{[27]}$

1. Moment one - before patient contact

2. Moment two - before aseptic task

3. Moment three - after body fluid exposure

4. Moment four - after patient contact

5. Moment five - after contact with patient surrounding

\section{Facemask}

Facemask is a barrier device used in infection control to prevent health care providers from breathing or coughing on patients.

Types of Facemask

1. Surgical or Medical mask

2. Surgical N95 mask

3. Homemade mask

4. $\quad$ Face covering

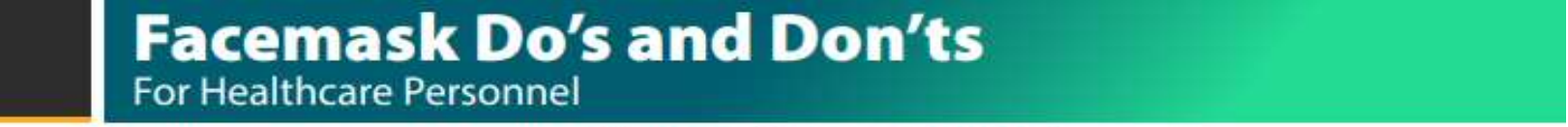

When putting on a facemask

Clean your hands and put on your facemask so it fully covers your mouth and nose.
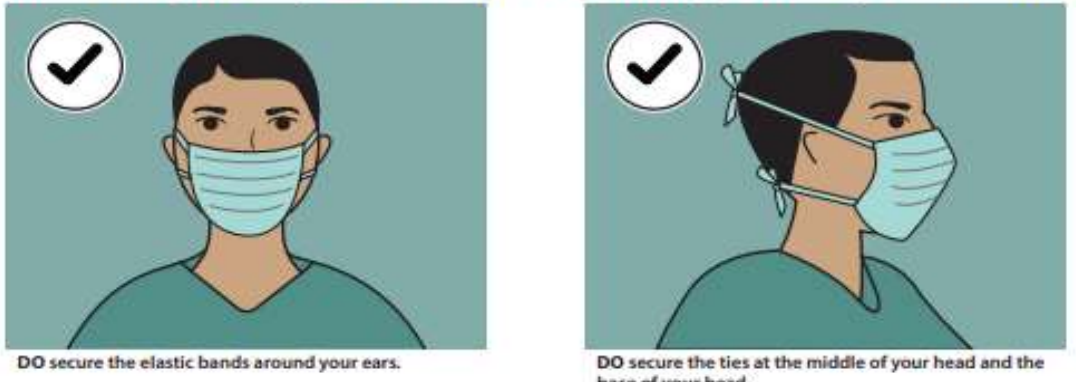

base of your head.

When wearing a facemask, don't do the following:
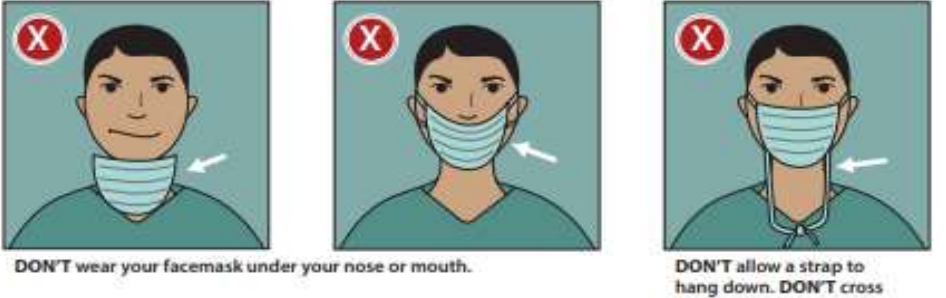

DON'T allow a strap to
hang down. DONT cross the straps.
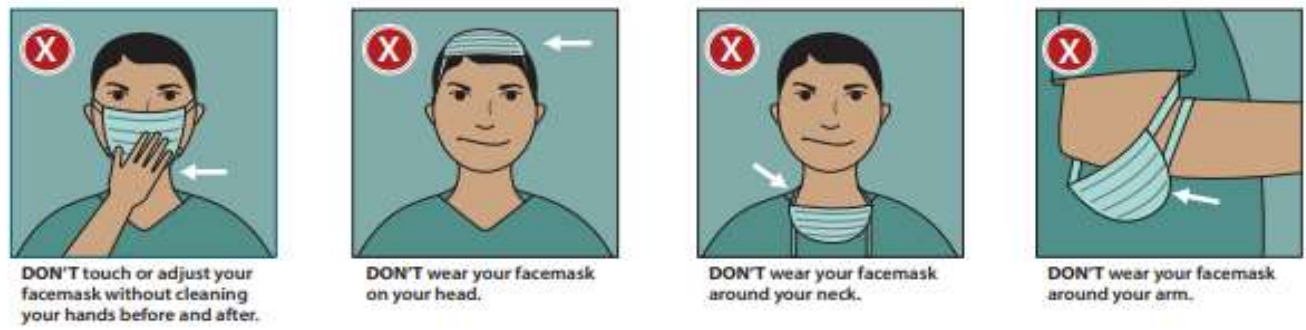

Proper hand hygiene should be followed strictly. Wash hands before putting the mask. Do not touch your mask while wearing it.If you touch your mask wash/ sanitize your hand immediately. Do not remove mask while talking to others. Do not use dirty mask, change often. 
When removing a facemask

Clean your hands and remove your facemask touching only the straps or ties.

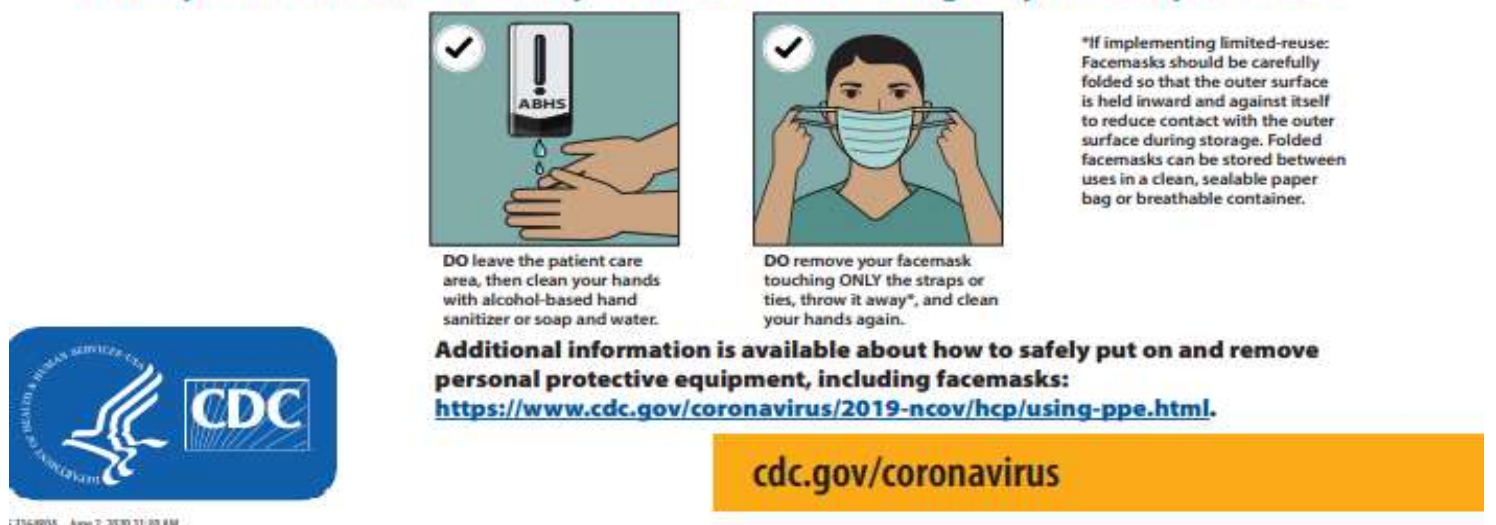

To remove mask, take straps and pull forward. Wash hands before and after removing mask. Place mask in a plastic bag. Reusable cloth mask should be wash with hot water and detergent,it should be completely dry before next use. Surgical mask should be discarded by following biomedical waste management.

\section{Respirator}

Respirator is a mask or device worn over the mouth and nose to protect the respiratory system by filtering out the dangerous substances from inhaled air.

\section{Parts of respirator}

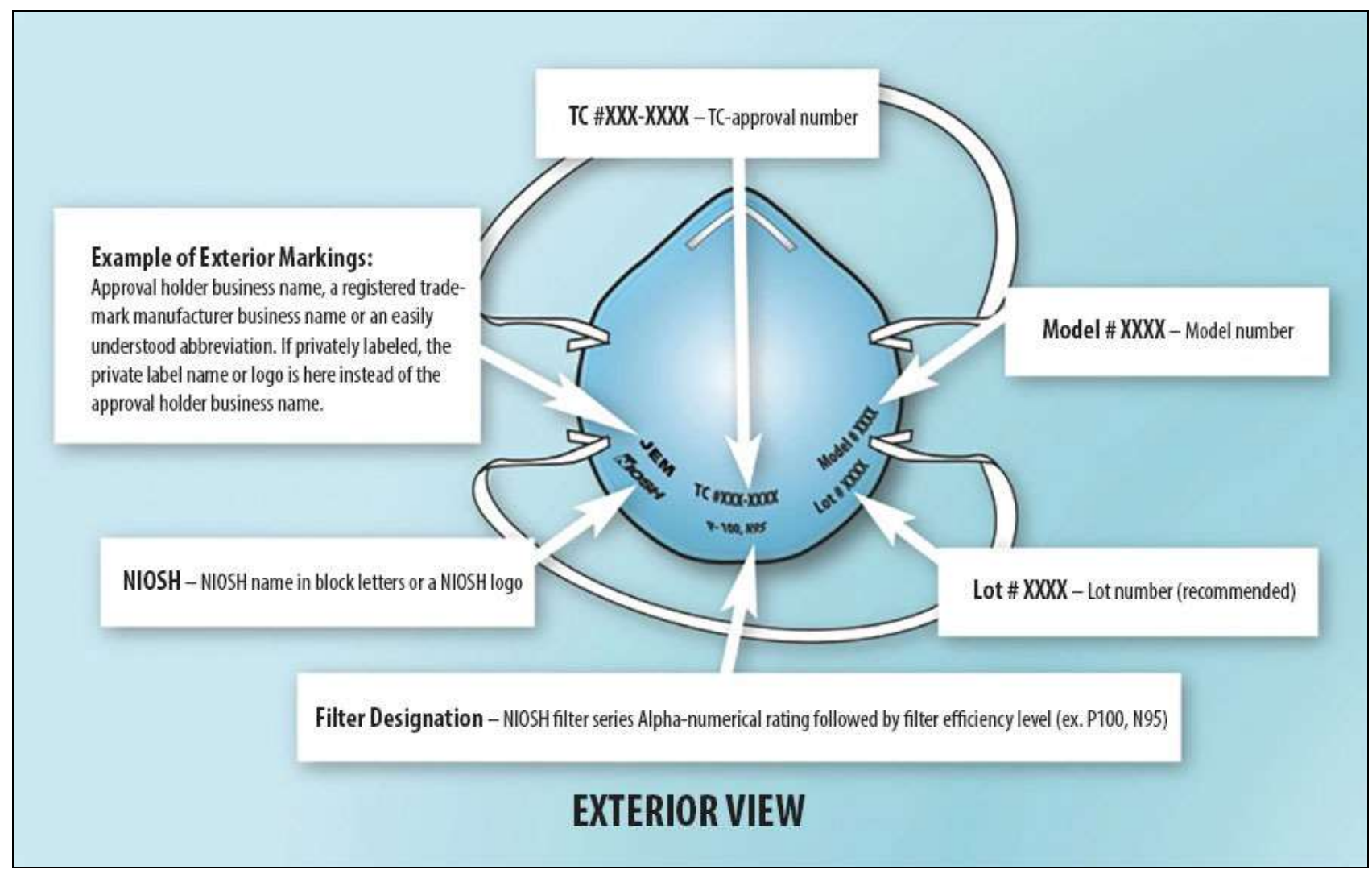

\section{Parts of respirator include}

1. Face piece,

2. Head straps,

3. Valves,

4. Connecting tubes,

5. Cartrideges,

6. Filters.

\section{Types of respirator}

1. Air- Purifying Respirators

2. Remove contaminants from the air

Particulate respirators 
Gas mask respirators

3. Air - supplying respirators

4. Provides clean source of air

a. Supplied air respirator

b. Self -contained breathing apparatus

\section{Select a type of respirator to see all approved models:}

N95 - Filters at least 95\% of airborne particles. Not resistant to oil. (N95 Manufacturers Index: $\underline{3 M} \underline{A} \underline{B} \underline{\subset} \underline{D} \underline{E} \underline{F} \underline{G} \underline{H} \underline{I} \mathrm{~J} \underline{K} \underline{L} \underline{M} \underline{N} \underline{O} \underline{P} \underline{Q} \underline{R} \underline{S}$ $\underline{I} \underline{U} \underline{V} \underline{W} \underline{X} \underline{Y} \underline{Z})$

Surgical N95 - A NIOSH-approved N95 respirator that has also been cleared by. the Food and Drug Administration (FDA) as a surgical mask.

N99-Filters at least 99\% of airborne particles. Not resistant to oil.

N100 - Filters at least $99.97 \%$ of airborne particles. Not resistant to oil.

R95 - Filters at least 95\% of airborne particles. Somewhat resistant to oil.

P95 - Filters at least $95 \%$ of airborne particles. Strong!y resistant to oil.

P99 - Filters at least $99 \%$ of airborne particles. Strong!y resistant to oil.

P100 - Filters at least $99.97 \%$ of airborne particles. Strongly resistant to oil.

\section{Guidelines for usage of respirator}

Inspect all parts of the respirator before use. The respirator must be put on correctly and worn during the exposure. The respirator must fit snugly against user's face to ensure that there are no gaps between thr user's skin and respirator seal. 


\section{Respirator On / Respirator Off}

\section{When you put on a disposable respirator}

Position your respirator correctly and check the seal to protect yourself from COVID-19.

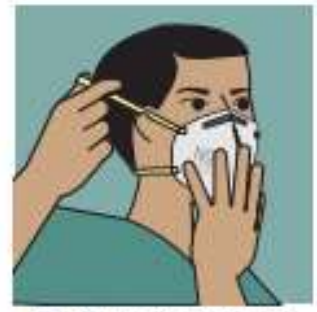

Cup the respirator in your hand. Hold the respinator under your chin wath the nose piece up. The top strap (on single or doub) strap respiratorsi goes over and rests at the top back of your head. The bottom strap is positioned around the neck and below the ears.

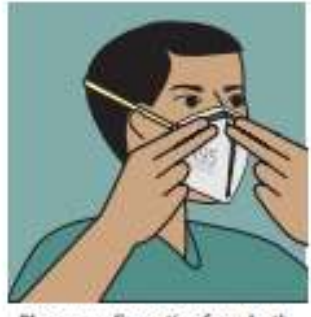

Place your fingertips from both hands at the top of the metal nose clip (if present). Slide fingertips down both sides of the metal strip to mold the nose area to the shape of your nose.

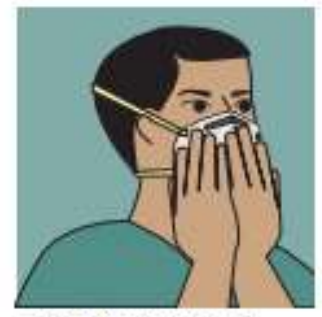

Place both hands over the respirator, take a quick breath in to check the seal. Breathe out. if pou feel a leak when breathing in or breathing out, there is not a proper seat.

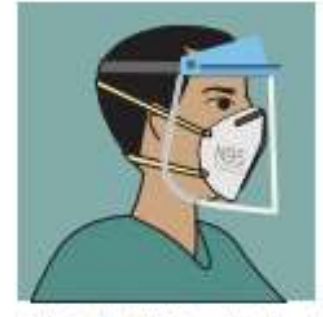

Select other PPE items that do nos interfere with tho fit of performance of your respirator.

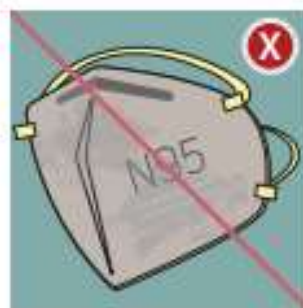

Do not use a respirator that appears damaged or deformed no longer forms an effective seal to the face, bocomes wet or visitbly dirty, or if breathing becomes difficult.

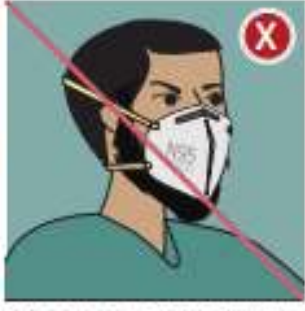

Do not allow tacial hair, jewolnys glasses, clothing, or anything etse to prevent proper placeme and the respirator.

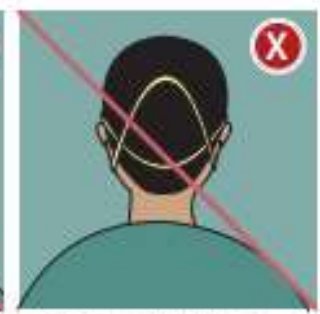

Do not crisscross the straps.

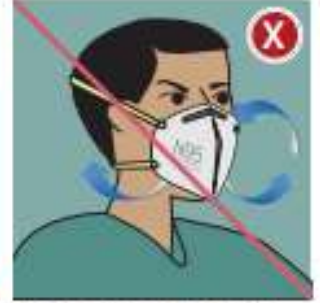

Do not wear a respirator that does not have a proper seal. in of try a different size or model.

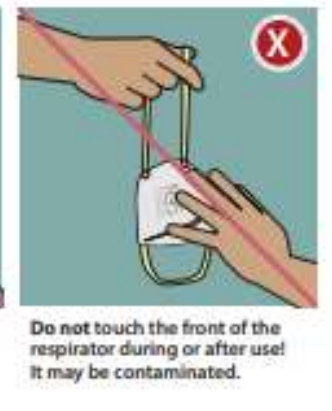

\section{When you take off a disposable respirator}

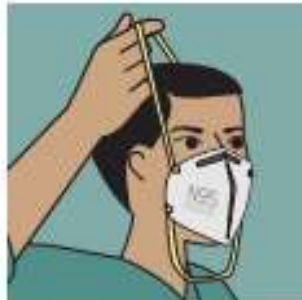

Remove by pulling the bottom strap over back of head, followed by the top strap, without touching the respiratov:

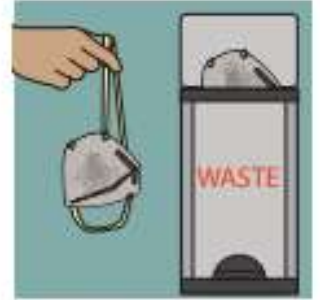

Discand in a waste container.

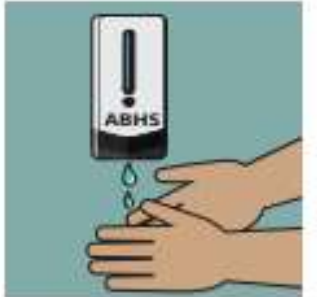

Clean your hands with alcohol based hand sanitizer or soap and water.

Employers must comply with the OSHA Respiratocy Protoction Standard, 29 CFR 1910.134, which includes medical evaluations, training and fit testing.

Additional information is avalable about how te safely put on and remeve personal protective equipment, including respirators:

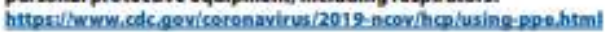

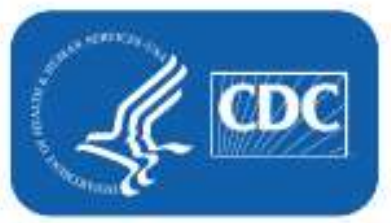

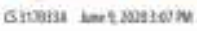

\section{cdc.gov/coronavirus}

Cleaning and disinfecting all the surfaces in clinic on a daily basis is a tough task and there may be chance of getting infection from fomites. So primary prevention is the better way to avoid getting infected. Hand Hygiene measures, social distancing norms, avoid frequent touching on facial surface should be practiced on a daily basis in this pandemic.

\section{Conclusion}

COVID-19 is a pandemic which puts both the patient and dentist at risk for cross infection Nevertheless, we cannot avoid dental practice during COVID -19 pandemic, because dental complaints may be an emergency. Nasal and or pharyngeal secretions are the prime source of infection and the dentist is exposed to this in close proximity during dental 
procedure. Dental procedures consisting of both aerosol and non-aerosol generating treatment adequate protection will be provided by dentist for patients following WHO and CDC guidelines. Dentist are advised to follow the standard guidelines and keep himself updated to protect his life and society. Research on infection control in the dental setting needs to be advanced.

\section{References}

1. Statement on the second meeting of the International health regulation. Emergency committee, WHO Director General's Statement on 30, January 2020, 2005.

2. Novel Coronavirus disease named COVID-19 on February 11, 2020, WHO.

3. Naming the Coronavirus disease COVID-19 and the virus causes it - WHO.

4. WHO Director. General's opening remarks at the media briefing on COVID-19, 11 March 2020.

5. Penghui Yang, Xiliang Wan. COVID-19: A new challenge for human beings Cellular \& Molecular Immunology, 2020.

https://doi.org/10.1038/s41423-020-0407-x

6. Family Coronaviridae, Viruses. DOI: http://dx.doi.org/10.1016/B978-0-12-803109-4.00017-9

7. Italy R Izzetti1, M Nisi1, M Gabriele1, F Graziani1 COVID-19. Transmission in dental practice: Brief Review of Preventive Measures in

8. LLM Poon, DKW Chu, KH Chan, OK Wong, TM Ellis, YHC Leung et al. Identification of a novel coronavirus in bats.

DOI: 10.1128/JVI.79.4.2001-2009.2005.

9. Jie Cui, ${ }^{* \dagger, 1}$ Naijian Han, ${ }^{\star, 1}$ Daniel Streicker, ${ }^{\S}$ Gang Li, * Xianchun Tang, * Zhengli Shi, ${ }^{\text {II }}$ Zhihong Hu, "I Guoping Zhao, \# Arnaud Fontanet, ${ }^{* *}$ Yi Guan, †'Linfa Wang, $\$$ Gareth Jones, ${ }^{\S}$ Hume E Field, $\mathbb{\Psi}$ Pet Per Daszak, 囚\#\# and Shuyi Zhang Evolutionary Relationships between Bat coronaviruses and their hosts. DOI: 10.3201/eid1310.070448

10. Background and summary of Novel coronavirus infection - as of 30 November 2012, WHO.

11. Coronavirus Disease 19 (COVID-19): Implications for Clinical Dental Care.

12. Gerardo Chowell, Fatima Abdirizak. Transmission characteristics of MERS and SARS in the Healthcare setting : a comparative study, September 2015,

13. Hamid Reza Fallahi, Seied Omid Keyhan, Dana Zandian, Seong-Gon Kim, Behzad Cheshmi. Being a front-line dentist during the COVID19 pandemic: A literature review.

14. Transmission of SARS-CoV-2: Implications for infection prevention precautions Scientific Brief 9 July 2020, WHO.

15. Ramanan Laxminarayan, Brian Wahl, Epidemiology and transmission dynamics of COVID-19 in two Indian States 2020.

16. Yonghyun Lee, Pokkee Min, Seonggu Lee. Shin - Woo Kim Prevalance and Duration of Acute loss of Smell or Taste in COVID-19 Patients,2020.

17. R Izzetti1, M Nisi1, M Gabriele, F Graziani. COVID-19 Transmission in dental practice: Brief review of preventive measures in Italy.

18. Laboratory testing for coronavirus disease (COVID-19) in suspected human cases Interim guidance 19 March WHO 2020,

19. Advice on the use of point-of care immunodiagnostic tests for COVID-19 Scientific Brief 8 April 2020, WHO.

20. Preeti Chawla Arora, Jasleen Kaur, Jasmine Kaur, Aman Arora. Teledentistry: An innovative tool for the underserved population

21. CDC Facilities COVID-19 Screening, Accessible version available at https://www.cdc.gov/screening/

22. Cleaning and disinfection of environmental surfaces in the context of COVID-19 Interim guidance 15 May 2020, WHO.

23. OSHA guidance summary: Dentistry workers and employers.

24. Considerations for the provision of essential oral health services in the context of COVID-19 Interim guidance 3 August 2020, WHO.

25. A Review Nisha Singh, Ankita Jain, Nidhi Sinha, Astha Chauhan, Rahila Rehman. Application of Four-Handed Dentistry in Clinical Practice.

26. Additional information is available about how to safely put on and remove personal protective equipment, including facemasks.

https://www.cdc.gov/coronavirus/2019-ncov/hcp/usingppe.html.COVID-19 Personal Protective Equipment (PPE) for Healthcare Personnel

27. Sax HABB, Allegranzi B, Uc I, Kay A, Larson BECJ, Boyce BDD, Pittet AB. My five moments for hand hygiene: a user-centred design approach to understand, train, monitor and report hand hygiene. 